\title{
Symmetric Functions and $q$-Products with an Application to Theoretical Physics
}

\author{
Maria E. X. Guimarães \\ Instituto de Fsica, Universidade Federal Fluminense, Niterói-RJ, Brazil \\ Email: emilia@if.uff.br, mexguimaraes@id.uff.br
}

How to cite this paper: Guimarães, M.E.X. (2021) Symmetric Functions and $q$-Products with an Application to Theoretical Physics. Advances in Pure Mathematics, 11, 386-394. https://doi.org/10.4236/apm.2021.115025

Received: April 9, 2021

Accepted: May 15, 2021

Published: May 18, 2021

Copyright (c) 2021 by author(s) and Scientific Research Publishing Inc. This work is licensed under the Creative Commons Attribution International License (CC BY 4.0).

http://creativecommons.org/licenses/by/4.0/

\begin{abstract}
The main purpose of this paper is to show that the Poincaré $q$-polynomials admit a representation in terms of the symmetric functions and the Patterson-Selberg (or Ruelle-type) spectral functions. We have shown that the $q$ series elliptic genera can be expressed in terms of $q$-analogs of the classical special functions, specially the equivalence between the spectral PattersonSelberg and the Ruelle functions. The main result of this manuscript is to show that this representation can be used in theoretical physics and we analyze them in terms of the Patterson-Selberg spectral function $\mathcal{R}(s)$.
\end{abstract}

\section{Keywords}

Poincaré $q$-Polynomials, Patterson-Selberg Spectral Functions, Ruelle Spectral Functions

\section{Introduction}

The main purpose of this paper is to present the explicit formulas of $q$-polynomials which admit a presentation in terms of the Patterson-Selberg (or Ruelle-type) spectral functions. It has been shown that equivariant methods also can be used to define and analyze (twisted) K-theory [1] [2] [3]. Applying techniques from the equivariant $\mathrm{K}$-theory, one can construct a Chern character and $\mathrm{K}$-theory Euler class. This is manifested by the fact that the $q$-series elliptic genera can be expressed in terms of $q$-analogs of the classical special functions, in particular the spectral Patterson-Selberg and Ruelle functions $\mathcal{R}(s)$ [4]. In the present paper we would like to call attention to this connection by discussing some mathematical aspects associated with it.

One of the aims of the present paper is the application of the analytic Patter- 
son-Selberg function $\mathcal{R}(s)$ and the symmetric functions (S-functions) in Analysis and Theoretical Physics. $\mathcal{R}(s)$ and S-functions not only provide beautiful formulas in analysis but also promote interesting results in theoretical physics. We mention below some results which will be explored along this manuscript.

Symmetric functions. Symmetric functions are directly connected with the Quantum Hall effect.

Finite-dimensional algebras. These algebras have wide variety of applications in Physics in the so-called quantum group which can be regarded as a deformation, depending on a parameter $q$, of the universal enveloping algebra of a semisimple Lie algebra. Therefore, this is not finite-dimensional algebras but they are finitely generated. Their representation theory for $q$ not a root of unity was found to be similar to the corresponding semi-simple Lie algebra.

Quantum groups. Quantum groups are an example of quasi-triangular Hopf algebras and, as such, for each quantum group there exists a universal R-matrix which intertwines with the action of the coproduct. By considering $q$-deformations of simple Lie superalgebras, one comes across to the concept of quantum supergroups.

Infinite-dimensional algebras. Of central importance in many diverse areas, Mathematics and Theoretical Physics are a particular class of infinite-dimensional algebras called (affine) Kac-Moody algebras. Unlike the finite-dimensional case, where the simple Lie algebras can be realized in terms of a finite number of fermionic/ bosonic modes, simple Kac-Moody algebras have various vertex operator realizations. As an example, in terms of a finite number of bosonic free fields, the modes of which generate a Heisenberg algebra.

This paper is organized as follows. In Section 2 we consider the twisted symmetric products, the Euler characteristic $\chi(X)$. Then in Section 3 we analyse the Hirzebruch $\chi\left(X^{n}\right)$ genera and calculate it in the orbifold cycle case. In Section 4 we calculate the orbifold symmetric product. In Section 5 we present the so-called Patterson-Selberg functions $\mathcal{R}(s)$ and discuss their applications. Finally, we summarize our key results in the Conclusion.

\section{The Twisted Symmetric Product}

Let $G$ be a finite group and $X$ be a $G$-space, on which a normal subgroup $A$ acts trivially. The $G$-equivariant K-theory of $X$ can be decomposed as a direct sum of twisted equivariant $\mathrm{K}$-theories of $X$ parametrized by the orbits of the conjugation action of $G$ on the irreducible representations of $A$ [5] (theorems 3.2 and 3.4).

Now we turn to the symmetric group and denote by $G=\mathfrak{S}_{n}$ the symmetric group on $n$ symbols. As before, we denote the non-trivial class of $\mathrm{G}$ by $\alpha$. Using the decomposition formula (theorem 3.4 of [5]), one can calculate $K_{\mathfrak{S}_{n}}^{*}\left(X^{n}, \alpha\right)$, where the group acts on the $n$-fold product of a manifold $X$ by permutation of coordinates. At last, formula for the twisted symmetric products takes the form 


$$
\begin{aligned}
& \sum q^{n} \chi\left(K_{\mathfrak{S}_{n}}^{*}\left(X^{n}, \alpha\right) \otimes \mathbb{C}\right) \\
& =\prod_{n>0}\left(1-q^{2 n-1}\right)^{-\chi(X)}+\prod_{n>0}\left(1+q^{2 n-1}\right)^{\chi(X)} \\
& \quad \times\left[1+\frac{1}{2} \prod_{n>0}\left(1+q^{2 n}\right)^{\chi(X)}-\frac{1}{2} \prod_{n>0}\left(1-q^{2 n}\right)^{\chi(X)}\right] .
\end{aligned}
$$

In Equation (1), $\chi(X)$ is the Euler characteristic of $X$ and $\left(^{*}\right)$ implies the conjugacy of the $K$ function. We can easily rewrite Equation (1) in terms of spectral functions $\mathcal{R}(s)$ and we will present it in section 5 .

It is known that if $X$ is any compact, connected manifold, then $\chi(X)=T d(X)$. By means of Atiyah-Singer formula one can calculate index of an elliptic operator and express it (i.e. express the signature of a manifold) in terms of Pontrjagin classes. As before, we denote the non-trivial class of $G$ by $\alpha$.

\section{The Analysis of the Hirzebruch Genera}

Let $M$ be a smooth manifold and $G$ is a finite group of diffeomorphisms. Note that there are two kinds of interesting cohomology theories for the orbifold $M / G$.

- The orbifold de Rham cohomology $H^{*}(M / G)$. We easily have isomorphism

$$
H^{*}(M / G) \cong H^{*}(M)^{G}
$$

- Next cohomology is the delocalized equivariant cohomology

$$
H^{*}(M, G)=\left(\bigoplus_{g \in G} H^{*}\left(M^{g}\right)\right)^{G}=\bigoplus_{[g] \in G_{*}}\left(M^{g}\right)^{Z_{g}}=\bigoplus_{[g] \in G_{*}} H^{*}\left(M^{g} / Z_{g}\right)
$$

Equations (3) have been introduced for the study of the equivariant K-theory. In this equations $G_{*}$ denotes the set of conjugacy classes of $G, Z_{g}$ denotes the centralizer of $g$. For this cohomology theory, one can define an Euler number, denoted by $\chi(M / G)$ and $\chi(M, G)$ respectively.

Definition 3.1

$$
\chi(M, G)=1 /|G| \sum_{(g, h)}^{\prime} \chi\left(M^{\langle g, h\rangle}\right)
$$

Here $\langle g, h\rangle$ is the group generated by $g$ and $h$, the sum is taken over commutating pairs $(g, h) \in G \times G$

Note that $\chi(M, G)$ as defined in (2) is the Euler number of $H^{*}(M, G)$ or equivalently $K_{G}^{*}(M)$. Therefore, one can easily show that

$$
\chi(M, G)=\sum_{(g, h)}^{\prime} \in G_{*} \chi\left(M^{g} / Z_{g}\right),
$$

it is a classical analogue of the Lefschitz formula. Indeed:

$$
\chi(M / G)=1 /|G| \sum_{g \in G} \chi\left(M^{g}\right)=\sum_{[g] \in G_{*}} 1 /\left|Z_{g}\right| \chi\left(M^{g}\right) .
$$


Interesting classes of examples are provided by the symmetric products $X^{(n)}=X^{n} / S_{n}$. The following formulas have been proved by various authors:

$$
\begin{aligned}
\sum_{n \geq 0} \chi\left(X^{(n)}\right) q^{n} & =1 /(1-q)^{\chi(X)} \\
\sum_{n \geq 0} \chi\left(X^{n}, S_{n}\right) q^{n} & =\prod_{l \geq 1} 1 /\left(1-q^{l}\right)^{\chi(X)}
\end{aligned}
$$

Again, we will present in Section 5, Equation (18), the result

$$
\sum_{n \geq 0} \chi\left(X^{(n)}\right) q^{n}=1 / \mathcal{R}\left(s=1 / 2 \pi i \tau\left(2-i \rho(\tau)-\frac{1}{2 \pi i \tau}\right)^{\chi(X)}\right.
$$

Analysis for cycles. Let $x_{j}, j=1, \cdots, d$ are the Chern roots of $X(d=\operatorname{dim} X)$.

If $\Delta_{n}(X)=(x, \cdots, x) \in X^{n}$ then notice $\left(X^{n}\right)^{\sigma_{n}}=\Delta_{n}(X),\left.X^{n}\right|_{\Delta_{n}(X)}$ is isomorphic to the direct sum of $\mathrm{n}$ copies of $X$ and $\sigma_{n}$ acts on it by cycling the factors $j$. The eigenvalues of $n$ cycles on $(\mathbb{C})^{n}$ are $\mathrm{e}^{(2 k \pi \sqrt{1 / n})}$ for $k=0, \cdots, n-1$ each with multiplicity 1.

$\prod_{k=1}^{n-1}\left[1-\mathrm{e}^{-x_{j}-2 k \pi(-1 / n)}\right]=\left(\prod_{k=1}^{\infty}-\prod_{k=n}^{\infty}\right)\left(1-\mathrm{e}^{-x_{j}-2 k \pi(-1 / n)}\right)$. Then with Appendix, Equation (18), we obtain

$$
\begin{aligned}
& \prod_{k=1}^{\infty}\left(1-\mathrm{e}^{-k x_{j}}\right)=\mathcal{R}\left(s=\left(1 / i \tau-x_{j}-1 / n\right)(1-i \rho(\tau))\right) \\
& \prod_{k=n}^{\infty}\left(1-\mathrm{e}^{-k x_{j}}\right)=\mathcal{R}\left(s=\left(n / i \tau-x_{j}-1 / n\right)(1-i \rho(\tau))\right)
\end{aligned}
$$

\section{The Orbifold Symmetric Product}

We will analyze the Poincaré polynomial of orbispace $\left[X^{n} / \mathfrak{S}_{n}\right]:=\left[X \times \cdots \times X / \mathfrak{S}_{n}\right][6]$, whose category objects are $n$-tuples $\left(x_{1}, \cdots, x_{n}\right)$ of points in $X$. In addition, the arrows are elements of the form $\left(x_{1}, \cdots, x_{n} ; \sigma\right)$, where $\sigma \in \mathfrak{S}_{n} .\left(x_{1}, \cdots, x_{n} ; \sigma\right)$ has its source as $\left(x_{1}, \cdots, x_{n}\right)$, while its target as $\left(x_{\sigma(1)}, \cdots, x_{\sigma(n)}\right) \cdot{ }^{1}$

The Poincaré $q$-polynomials. Let $X$ be a topological space, denoted by $P(X, y)$ its Poincaré polynomial, $P(X, y)=\sum_{j} b^{j}(X) y^{j}$. Here $b^{j}(X)$ is the $j$-th Betti number of $X$. The following formula has been proved by Macdonald [7]

$$
\sum_{n=0}^{\infty} \mathrm{P}\left(X^{n} / \mathfrak{S}_{n}, y\right) q^{n}=\frac{\prod_{j}\left(1+Q y^{2 j+1}\right)^{b^{2 j+1}(X)}}{\prod_{j}\left(1-Q y^{2 j}\right)^{b^{2 j}(X)}} .
$$

For $y=-1$, Equation (12) reduces to the formula for the Euler characteristic of the symmetric product $\sum_{n=0}^{\infty} \chi\left(X^{n} / \mathfrak{S}_{n}\right) Q^{n}=(1-Q)^{-\chi(X)}$, which is valid for ${ }^{1}$ The mentioned category is a groupoid for the inverse of $\left(x_{1}, \cdots, x_{n} ; \sigma\right)$, which is $\left(x_{1}, \cdots, x_{n} ; \sigma^{-1}\right)$. This is the reason why we can consider $\left[X^{n} / \mathfrak{S}_{n}\right]$ as an orbispace. 
the topological space $X$ whose cohomology $H^{j}(X$; real $)$ is finitely generated for each $j \geq 0$. Using the $\mathfrak{S}_{n}$-equivariant K-theory of $X^{n}$, one can arrive at the following formula for generation functions [6]:

$\sum_{n=0}^{\infty} \chi_{\mathfrak{S}_{n}}\left(X^{n} / \mathfrak{S}_{n}\right) q^{n}=\prod_{j>0}\left(1-Q^{j}\right)^{-\chi(X)}$. In terms of the spectral functions $\mathcal{R}(s)$, Equation (12) takes the form

$$
\begin{aligned}
& \sum_{n=0}^{\infty} \mathrm{P}\left(X^{n} / \mathfrak{S}_{n}, y\right) q^{n} \\
& =\frac{\mathcal{R}(s=(1-i(\log (y))(2 j+1) /(2 \pi \tau))(1-i \rho(\tau))+i \sigma(\tau))^{b_{2 j+1}(X)}}{\mathcal{R}\left(s=(1-i(\log (y))(j / \pi \tau))(1-i \rho(\tau))^{b_{2 j}(x)}\right.} .
\end{aligned}
$$

The Poincaré orbifold polynomial can be defined as follows [6]

$$
\mathrm{P}_{\text {orb }}([X / G], y):=\sum y^{j} \operatorname{rank} H_{\text {orb }}^{j}([X / G] \text {; real }) \equiv \sum y^{j} b_{\text {orb }}^{j}([X / G]),
$$

where $b_{\text {orb }}^{j}$ is the $j$-th orbifold Betti number.

Remark 4.1 For any arbitrary (fixed) positive integers $m_{1}, \cdots, m_{r}$ the following equality holds

$$
\sum_{n_{1}, \cdots, n_{r}=0}^{\infty} F\left(n_{1}, \cdots, n_{r}\right) z_{1}^{n_{1}}, \cdots, z_{r}^{n_{r}}=\sum_{N=0}^{\infty} \sum_{\substack{n_{1} m_{1}+\cdots, n_{n} m_{r}=N \\ n_{1}, \cdots, n_{r} \geq 0}} F\left(n_{1}, \cdots, n_{r}\right) z_{1}^{n_{1}}, \cdots, z_{r}^{n_{r}} .
$$

For the symmetric product, viewed as an orbifold groupoid $\left[X^{n} / \mathfrak{S}_{n}\right]$, it follows that

$$
H_{\text {orb }}^{*}\left(\left[X^{n} / \mathfrak{S}_{n}\right] ; \text { real }\right) \cong \underset{\sum j n_{j}=n}{\bigoplus} \underset{j}{\bigotimes} H^{*}\left(X^{n_{j}} ; \text { real }\right)^{\mathfrak{S}_{n_{j}}} .
$$

Using Equation (15) for calculating the orbifold Poincaré polynomial, we obtain

$$
\begin{aligned}
& \sum_{n=0}^{\infty} q^{n} \mathrm{P}_{\text {orb }}\left(\left[X^{n} / \mathfrak{S}_{n}\right], y\right) \\
& =\sum_{n=0}^{\infty} q^{n}\left(\sum_{\sum n_{j}=n} \prod_{j} \mathrm{P}\left(X^{n_{j}} / \mathfrak{S}_{n_{j}}, y\right)\right) \\
& =\prod_{j>0} \prod_{n \geq 0} \frac{\left(1+q^{n} y^{2 j+1}\right)^{b^{2 j+1}(X)}}{\left(1-q^{n} y^{2 j}\right)^{b^{2 j}(X)}} \\
& =\prod_{j>0} \frac{[\mathcal{R}(s=-i(2 j+1) \log (y) /(2 \pi \tau)(1-i \rho(\tau))+i \sigma(\tau))]^{2^{j+1}(X)}}{\left[\mathcal{R}(s=-i j \log (y) /(\pi \tau)(1-i \rho(\tau))]^{b^{2 j}(X)}\right.} .
\end{aligned}
$$

For these formulas the cohomology of $X$ must be finitely generated at each $n$. The first line in (17) is similar to the equality (2.32) of [6]. As before $b^{j}(X)$ is the $j$-th Betti number of $X$.

\section{The Ruelle-Type Spectral Functions}

Let us introduce the Ruelle-type spectral function of a hyperbolic geometry $\mathcal{R}(s)$ [4]. The function $\mathcal{R}(s)$ is an alternating product of more complicated 
factors, each one being the so-called Patterson-Selberg function,

$$
\begin{gathered}
\prod_{n=\ell}^{\infty}\left(1-q^{a n+\varepsilon}\right)=\mathcal{R}(s=(a \ell+\varepsilon)(1-i \rho(\tau))+1-a), \\
\prod_{n=\ell}^{\infty}\left(1+q^{a n+\varepsilon}\right)=\mathcal{R}(s=(a \ell+\varepsilon)(1-i \rho(\tau))+1-a+i \sigma(\tau)),
\end{gathered}
$$

where $q \equiv \mathrm{e}^{2 \pi i \tau}, \quad \rho(\tau)=\operatorname{Re} \tau / \operatorname{Im} \tau, \quad \sigma(\tau)=(2 \operatorname{Im} \tau)^{-1}, \quad a$ is a real number, $\varepsilon, b \in \mathbb{C}, \quad \ell \in \mathbb{Z}_{+}$. The readers can find explicit formula for $\mathcal{R}(s)$ and its analytic properties in the reference [4].

The Bailey's Transform and Infinite Hierarchy of the Bailey's Chain

Let us begin with notations: $(a ; q)_{\infty}:=\prod_{m=0}^{\infty}\left(1-a q^{m}\right)$, and $(a ; q)_{n}:=(a ; q)_{\infty} /\left(a q^{n} ; q\right)_{\infty}$. Bailey made the following observation [8] (see also [9]), which is known as Bailey's transform.

Theorem 5.1 (lectures [9], Theorem 3.1) Let for a suitable convergence conditions,

$$
\begin{aligned}
& \beta_{n}=\sum_{r=0}^{n} \alpha_{r} u_{n-r} v_{n+r}, \\
& \gamma_{n}=\sum_{r=n}^{\infty} \delta_{r} u_{r-n} v_{r+n},
\end{aligned}
$$

then

$$
\sum_{n=0}^{\infty} \alpha_{n} \gamma_{n}=\sum_{n=0}^{\infty} \beta_{n} \delta_{n}
$$

Proof.

$$
\sum_{n=0}^{\infty} \alpha_{n} \gamma_{n}=\sum_{n=0}^{\infty} \sum_{r=n}^{\infty} \alpha_{n} \delta_{r} u_{r-n} v_{r+n}=\sum_{r=0}^{\infty} \sum_{n=0}^{r} \alpha_{n} \delta_{r} u_{r-n} v_{r+n}=\sum_{r=0}^{\infty} \delta_{r} \beta_{r} .
$$

Important application of the Bailey's transform and Bailey's Lemma can be found in [8], Section 4

Let for $n \geq 0$

$$
\beta_{n}=\sum_{r=0}^{n} \frac{\alpha_{r}}{(q ; q)_{n-r}(a q ; q)_{n+r}}
$$

then also

$$
\beta_{n}^{\prime}=\sum_{r=0}^{n} \frac{\alpha_{r}^{\prime}}{(q ; q)_{n-r}(a q ; q)_{n+r}}
$$

In addition

$$
\begin{gathered}
\alpha_{r}^{\prime}=\alpha_{r} \frac{\left(\rho_{1} ; q\right)_{r}\left(\rho_{2} ; q\right)_{r}\left(a q / \rho_{1} \rho_{2}\right)^{r}}{\left(a q / \rho_{1} ; q\right)_{r}\left(a q / \rho_{2} ; q\right)_{r}} \\
\beta_{n}^{\prime}=\sum_{r \geq 0} \beta_{r} \frac{\left(\rho_{1} ; q\right)_{r}\left(\rho_{2} ; q\right)_{r}\left(a q / \rho_{1} \rho_{2} ; q\right)_{n-r}\left(a q / \rho_{1} \rho_{2}\right)^{j}}{(q ; q)_{n-j}\left(a q / \rho_{1} ; q\right)_{n}\left(a q / \rho_{2} ; q\right)_{n}} .
\end{gathered}
$$

We apply Bailey's transform

$$
u_{n-r}:=\prod_{m=0}^{n-r}\left(1-q^{m+1}\right) \equiv(q ; q)_{n-r}=\frac{(q ; q)_{\infty}}{\left(q q^{n-r} ; q\right)_{\infty}}=\prod_{m=0}^{\infty} \frac{1-q^{m+1}}{1-q^{m+n-r+1}}
$$




$$
\begin{gathered}
=\frac{\mathcal{R}(s=1-i \rho(\tau))}{\mathcal{R}(s=(n-r+1)(1-i \rho(\tau)))}, \\
v_{n+r}:=\prod_{m=0}^{n+r}\left(1-a q^{m+1}\right) \equiv(a q ; q)_{n+r}=\frac{(q ; q)_{\infty}}{\left(a q^{n+r} ; q\right)_{\infty}}=\prod_{m=0}^{\infty} \frac{1-q^{m+1}}{1-a q^{m+n+r+1}} \\
=\frac{\mathcal{R}(s=1-i \rho(\tau))}{\mathcal{R}(s=(n+r+\xi+1)(1-i \rho(\tau)))},
\end{gathered}
$$

where $\xi=\log a / 2 \pi i \tau$.

Using Equations (29) and (31) we have:

$$
\begin{aligned}
& \beta_{n}=\sum_{r=0}^{n} \alpha_{r} \frac{\mathcal{R}(s=(n-r+1)(1-\rho(\tau))) \cdot \mathcal{R}(s=(n-r+1)(1-\rho(\tau)))}{\mathcal{R}(s=1-i \rho(\tau)) \cdot \mathcal{R}(s=1-i \rho(\tau))}, \\
& \beta_{n}^{\prime}=\sum_{r=0}^{n} \alpha_{r}^{\prime} \frac{\mathcal{R}(s=(n-r+1)(1-\rho(\tau))) \cdot \mathcal{R}(s=(n-r+1)(1-\rho(\tau)))}{\mathcal{R}(s=1-i \rho(\tau)) \cdot \mathcal{R}(s=1-i \rho(\tau))} .
\end{aligned}
$$

In addition (see [9]),

$$
\begin{gathered}
\alpha_{r}^{\prime}=\alpha_{r} \frac{\left(\rho_{1} ; q\right)_{r}\left(\rho_{2} ; q\right)_{r}\left(a q / \rho_{1} \rho_{2}\right)^{r}}{\left(a q / \rho_{1} ; q\right)_{r}\left(a q / \rho_{2} ; q\right)_{r}}, \\
\beta_{n}^{\prime}=\sum_{r \geq 0} \beta_{r} \frac{\left(\rho_{1} ; q\right)_{r}\left(\rho_{2} ; q\right)_{r}\left(a q / \rho_{1} \rho_{2} ; q\right)_{n-r}\left(a q / \rho_{1} \rho_{2}\right)^{r}}{(q ; q)_{n-j}\left(a q / \rho_{1} ; q\right)_{n}\left(a q / \rho_{2} ; q\right)_{n}} .
\end{gathered}
$$

Important special cases of Bailey's Lemma and iterated of these results where found in [9]. Note that a pair of sequences $\left(\alpha_{n}, \beta_{n}\right)$ is called a Bailey pair. Thus if $\left(\alpha_{n}, \beta_{n}\right)$ is a Bailey pair, then $\left(\alpha_{n}^{\prime}, \beta_{n}^{\prime}\right)$ is a new pair which is given by (34) and (35).

Bailey's Chain. Using Bailey initial pair $\left(\alpha_{n}^{\prime}, \beta_{n}^{\prime}\right)$ we can create a new pair $\left(\alpha_{n}^{\prime \prime}, \beta_{n}^{\prime \prime}\right)$ by applying Bailey's Lemma. Continuing this process we create a sequence of Baley pairs, Bailey's chain:

$$
\left(\alpha_{n}, \beta_{n}\right) \rightarrow\left(\alpha_{n}^{\prime}, \beta_{n}^{\prime}\right) \rightarrow\left(\alpha_{n}^{\prime \prime}, \beta_{n}^{\prime \prime}\right) \rightarrow\left(\alpha_{n}^{\prime \prime \prime}, \beta_{n}^{\prime \prime \prime}\right) \rightarrow \cdots
$$

Let us assume $\left(\alpha_{n}, \beta_{n}\right)=\left(\alpha_{n}^{0}, \beta_{n}^{0}\right)$, then this allows to extend the Bailey chain (36) to the left [9]:

$$
\cdots \rightarrow\left(\alpha_{n}^{(-2)}, \beta_{n}^{(-2)}\right) \rightarrow\left(\alpha_{n}^{(-1)}, \beta_{n}^{(-1)}\right) \rightarrow\left(\alpha_{n}^{0}, \beta_{n}^{0}\right) \rightarrow\left(\alpha_{n}^{\prime}, \beta_{n}^{\prime}\right) \rightarrow \cdots
$$

A Bailey pair is uniquely determined given either sequence $\alpha_{n}$ or $\beta_{n}$. Let a sequence $\alpha_{n}$ be given, then $\beta_{n}$ sequence can be found. Equation (24) and Equation (25) can be inverted, and the result is [9]:

$$
\begin{aligned}
\alpha_{n} & =\left(1-a q^{2 n}\right) \sum_{k=0}^{n} \beta_{k} \frac{(a q ; q)_{n+k-1}(-1)^{n-k} q^{C_{2}^{n-k}}}{(q ; q)_{n-k}} \\
& =\left(1-a q^{2 n}\right) \sum_{k=0}^{n} \beta_{k} \frac{\prod_{m=0}^{\infty}\left(1-a q^{m+n+k}\right)(-1)^{n-k} q^{C^{n-k}}}{\prod_{m=0}^{\infty}\left(1-q^{m+n-k+1}\right)} .
\end{aligned}
$$

Finally, we obtain 


$$
\alpha_{n}=\left(1-a q^{2 n}\right) \sum_{k=0}^{n} \beta_{k} \frac{\mathcal{R}(s=(n+k)(1-i \rho(\tau)))(-1)^{n-k} q^{C_{2}^{n-k}}}{\mathcal{R}(s=(n-k+1)(1-\rho(\tau)))} .
$$

\section{Conclusion}

The main purpose of this paper is to present an explicit formula of $q$-polynomials which admit presentation in terms of the Patterson-Selberg (or Ruelle-type) spectral functions. Equivariant methods also can be used to define and analyze (twisted) K-theory. Applying techniques from the equivariant K-theory, one can construct a Chern character and K-theory Euler class. This is manifested by the fact that the $q$-series elliptic genera can be expressed in terms of $q$-analogs of the classical special functions, in particular the spectral Patterson-Selberg and Ruelle functions $\mathcal{R}(s)$. Our main results were presented in Section 5 and they were applied them in some interesting problems in theoretical physics.

\section{Acknowledgements}

The author would like to thank Prof. A. A. Bytsenko for fruitful discussions during the preparation of this manuscript.

\section{Conflicts of Interest}

The author declares no conflicts of interest regarding the publication of this paper.

\section{References}

[1] Adem, A. and Ruan, Y. (2003) Twisted Orbifold K-Theory. Communications in Mathematical Physics, 237, 533-556. https://doi.org/10.1007/s00220-003-0849-x

[2] Wang, W. (2003) Equivariant K-theory, Generalized Symmetric Products and Twisted Heisenberg Algebra. Communications in Mathematical Physics, 234, 101-127. https://doi.org/10.1007/s00220-002-0753-9

[3] Freed, D.S., Hopkins, M.J. and Teleman, C. (2008) Twisted Equivariant K-Theory with Complex Coefficients. Journal of Topology, 1, 16-44. https://doi.org/10.1112/jtopol/jtm001

[4] Bonora, L. and Bytsenko, A. A. (2011) Partition Functions for Quantum Gravity, Black Holes, Elliptic Genera and Lie Algebra Homologies. Nuclear Physics B, 852, 508-537. https://doi.org/10.1016/j.nuclphysb.2011.07.002

[5] Gómez, J.M. and Uribe, B. (2007) A Decomposition of Equivariant K-Theory in Twisted Equivariant K-Theories. International Journal of Mathematics, 28, Article ID: 1750016. https://doi.org/10.1142/S0129167X17500161

[6] Lupercio, E., Uribe, B. and Xicotencatl, M.A. (2007) The Loop Orbifold of the Symmetric Product. Journal of Pure and Applied Algebra, 211, 293-306. https://doi.org/10.1016/j.jpaa.2007.01.002

[7] Macdonald, I.G. (1962) The Poincaré Polynomial of a Symmetric Product. Mathematical Proceedings of the Cambridge Philosophical Society, 58, 563-568. https://doi.org/10.1017/S0305004100040573

[8] Bailey, W.N. (1947) Some Identities in Combinatory Analysis. Proceedings of the 
London Mathematical Society, 49, 421-435.

https://doi.org/10.1112/plms/s2-49.6.421

[9] Andrews, G.E. (1985) $q$-Series: Their Development and Application in Analysis, Number Theory, Combinatorics, Physics, and Computer Algebra. Regional Conference in Mathematics, vol. 66, Published by the American Mathematical Society, Providence, Rhode Island. https://doi.org/10.1090/cbms/066 